\title{
SMART ASSIMILATION OF RENEWABLE BASED MICROGRIDS
}

\author{
Prajith Prabhakar, Muthu kumari T, Senthil Kumar C, P. Selvaraj
}

\begin{abstract}
Expansion from the existing power transmission and distribution with close coactions with energy, control and communication is possible with the help of Smart grid. Microgrid plays an important part in the coalition of this integration of smart grid. Using both kinds of energy systems a proximity with the loads and actuation using power electronic devices and systems can be achieved with power processing. There is a challenge in such type of interactive, buoyant and sustainable model. In this paper, a unique modeling and control prototype is conferred for the integration of renewable based Microgrid into the smart grid. More renewable energy resources can be added into the Microgrid considering the design requirements. The proposed model versatility is experimented in MATLAB/ Simulink platform to demonstrate the transition of states in Microgrid and overall power conditioning with transient response of the system.

Index terms - Smart grid, Microgrid, Integration, power conditioner, Renewable Energy.
\end{abstract}

\section{INTRODUCTION}

Modernized power system is a collection of more number the main stream with a criterion to improve efficiency, reliability and also to reduce cost [1]. This system consists of small power sources which is called as the building blocks such as solar, wind, fuel cells etc. The very advanced way to connect these resources is by a structure called Microgrid. The advancement in the technologies carries out power electronic devices and interfaces, information and communication and supervisory control systems. For the electric power distribution and storage purposes, nonconventional energy sources and systems (RESS) like biomass, biogas, geothermal etc plays a significant role. Resilient energy infrastructure idea minimizes the cost for remote power and green like Microgrid and sustainable development efforts [3]. The restoration of power quality complications are observed with the integration small and co-located Microgrid with renewable energy resources into the existing conventional Grid [4]. Lower the environmental impacts and improvement of security of supply, all of these are combined in the form of distributed generation based Microgrid. A realistic model of this type from the advanced research methodologies in the area of Microgrid paved the way to the complex and hybrid power generation, supply and storage system which must be robust and sustainable.

Revised Manuscript Received on August 19, 2019.

Prajith Prabhakar, Assistant Professor/EEE, Jeppiaar Institute of Technology, Chennai, Tamil Nadu, India.

Muthu kumari. T, Jeppiaar Institute of Technology, Chennai, Tamil Nadu, India.

Senthil Kumar C, Jeppiaar Institute of Technology, Chennai, Tamil Nadu, India.

P. Selvaraj, Professor/EEE, Sri Venkateswara Engineering College, Tirupathi, AP, India. of energy sources. The coagulation of these resources within

Smart grid archetype uses information and communications (ICT) collects information and actuates all processes which improve resilience, power quality, sustainability for the production of electricity [5]. More attention are given to the energy systems and communication networks due to the supplies of urban services. Utilities use several information technologies for the support of optimum performance and uninterruptible power supply to the Microgrid. For the efficient balance of energy demand and supply to the Microgrid due to the increase in urbanization demand, there is a rise in usage of renewable technologies. On the other side, continuous and independent formation of renewable energy sources and systems creates risks in terms of voltage and power fluctuations and other critical issues. With the smart grid concept in Microgrid enables real time optimization and interaction with other facilities help of integration of distributed energy sources [7]. For the development of smart cities, grid integration of renewable resources plays a crucial role with all technical implications in Microgrid.

In the Microgrid based system, various sources at watts and $\mathrm{kW}$ level acts as essential part of power generation and distribution along -with the main energy sources. Microgrid along with the distributed generation performs the concern operation in grid- synchronized or isolated modes. Established efficiency and cost effectiveness, for providing uninterruptable supply to the loads from the sources, several combinations can be made on the operation wise. Consideration of all diverse factors in physical components and also the decentralized communication between the units, modeling of such system will be complex with unstable nature of RES and transient power quality conditions [8]. A combined modeling approach is been proposed in this paper that manage reliable and more efficient smart grid based Microgrid in different operating states. The transient response of the proposed system was validated using a fault in the Microgrid by the approach. Three phase fault is given to the proposed system and with the help of smart controller, monitors the variation in reactive power and harmonics. The next section describes the proposed system in Microgrid, following by system integration and control with experimental analysis section and finally the conclusion.

\section{PROPOSED DESIGN}

A smart Microgrid mainly consists of smart controller that controls the complete system in a smart way. An enriched smart Microgrid system is shown in the Figure 2.1. 
Smart grid model with incorporation of renewable energy based microgrid and also the generic components are shown in the diagram below.

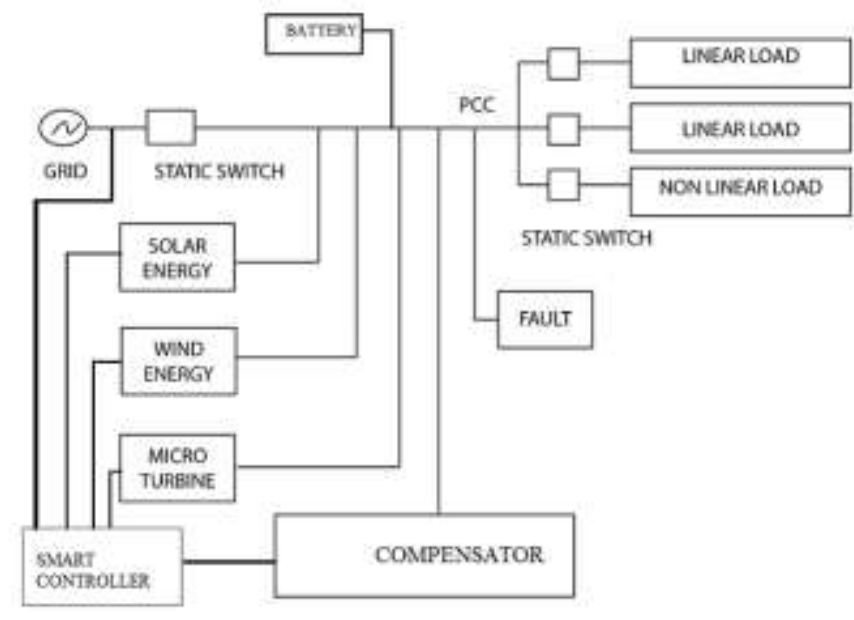

Fig.2.1. Smart controller within Microgrid.

The main utility grid which serves as a primary grid in the form of large generator and its excitation system and control are done with the excellent smart controller in a methodological way. This supplies primary power through the conventional transmission line. On the other side, renewable energy resources along with the respective power electronic converters and interfaces are connected to the AC load bus using static or dynamic coupling. During dynamic actions, a continuation in the power supply is provided by the supervisory control scheme in Microgrid smart central controller [MSCC]. Considering black out or service hours on the main grid side or transmission lines, the switching take place with the help of Predictive technique in MSCC and continues the power supply through RESs. In any dynamic conditions Microgrid keep operating with synchronization with main grid.

It is apparent from the figure 2.1 that distributed power system based Microgrid comprises of different energy sources that provides power, Voltage source inverter or converter and coupling equipment or link that connects the microgrid to the primary grid. The toolbox is available in MATLAB/ Simulink platform, Simpower systems for each and every component. The analysis and modeling of every component can do with these libraries. The analysis of complete proposed system behavior including transient, harmonic (THD), power flow and other all types of key in power system and automation is possible with the available libraries in Simpower system.

The challenges in the integration of renewable sources into the main grid by means of Static switch can be overcome with the latest technologies available in the present day. Microgrids are small scale electrical power generation and distribution systems interconnecting with the primary conventional grid in all means [9]. For the improvement of resilience, efficiency and transient response of the smart grid system approaches like cyber physical design can be used in the proposed system [10]-[12]. In model predictive method, using dynamic matrix control design scattered and hybrid power sources and their combinations will be controlled along with various electrical parameters. Programming is possible with MSCC to add the most economical power sources or efficient and reliable combinations into the Microgrid due to the demand of power and excessive can be dumped into the storage devices. The components needed for the assimilation are described in subsections:

\section{A. Micro-sources}

A small power source of energy which is integrated into the main microgrid ac bus with the help of converters is known as micro- sources. Renewable energy sources are usually used as micro- sources. High efficiency, less pollution and flexibility are the advantages of the wind, solar energy and Micro turbines. With these add-ons Microgrid can be constituted very reliably to form hybrid power system [12]. Figure 2.3 and 2.4 shows the model for wind and solar from Simpower systems tool box that can be used as micro-sources.

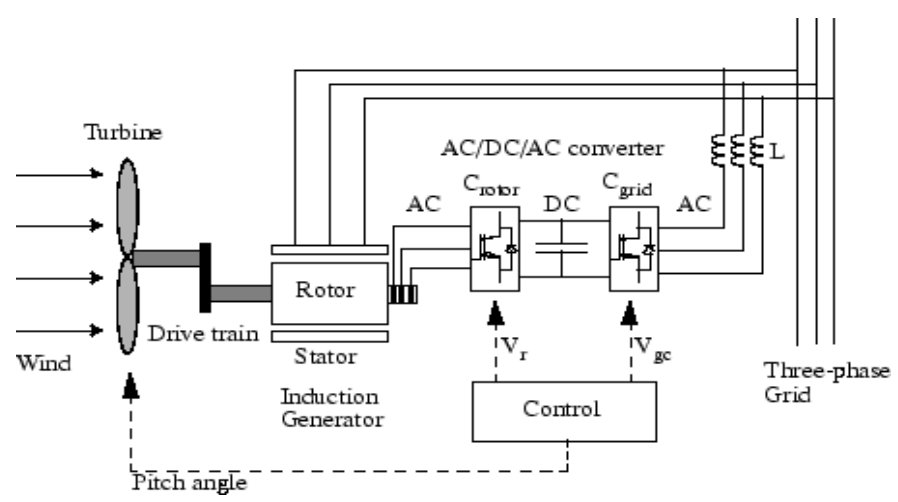

Figure 2.2. Wind Model to Microgrid

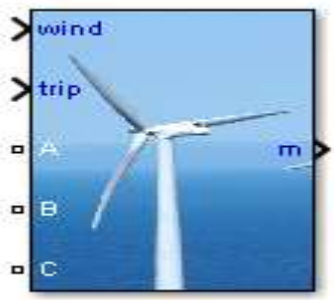

Figure 2.3. Wind MATLAB Model

The above figure shows the MATLAB version of wind turbine model used in MATLAB/ Simulink 2019a can be used as micro- sources.

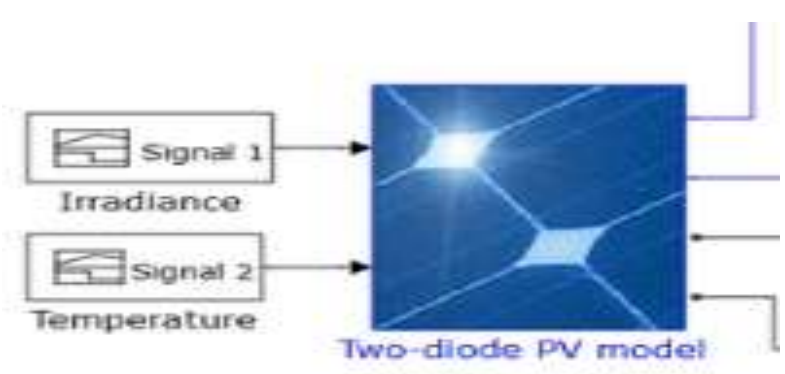

Figure 2. 4. Solar MATLAB model

Published By: Blue Eyes Intelligence Engineering 
The predictive model for solar and wind will be used in the integration into the Microgrid and main grid. Synchronization of the microgrid resources with primary grid after the assimilation of renewable with the help of model predictive technique is shown below.

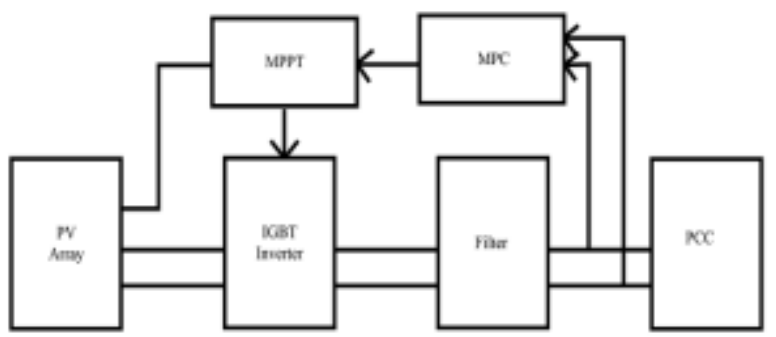

Figure 2.5. Predictive model for solar Energy

The Microturbine predictive model is shown in the figure 2.6. Smart integration of the microgrid with the help of predictive technique is used in MSCC.

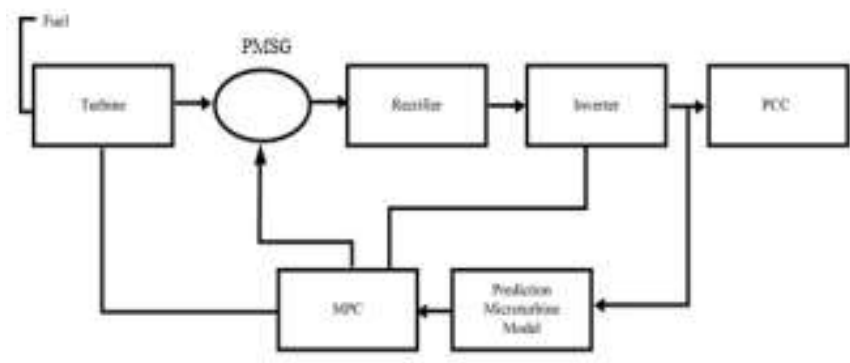

Figure 2.6. Microturbine model

The generic implementation of Solar cell, Wind turbines and also Microhydel type turbines are parameterized that corresponds to attractive types of bundles which can use in Microgrid.

\section{B. Voltage Source Inverter}

When connection comes for the micro- source to the main ac load bus, inverter comes into the scene. With the help of proper control method and pulses from the Microgrid smart central controller the modeled RES can be connected into the Grid. Since the MSCC are modeled with predictive technique, it helps in the convenient connection of the distributed generators into the load bus in both grid synchronized as well as Isolated mode of Microgrid operation. Individual model of VSI comprises of DG source, inverter and proper filter for the production of output sine wave along with predictive controller. The Solar cell/PV array, Wind and Microturbine based predictive model with VSI can be developed form the MATLAB platform which is demonstrated in the figure 2.7 .

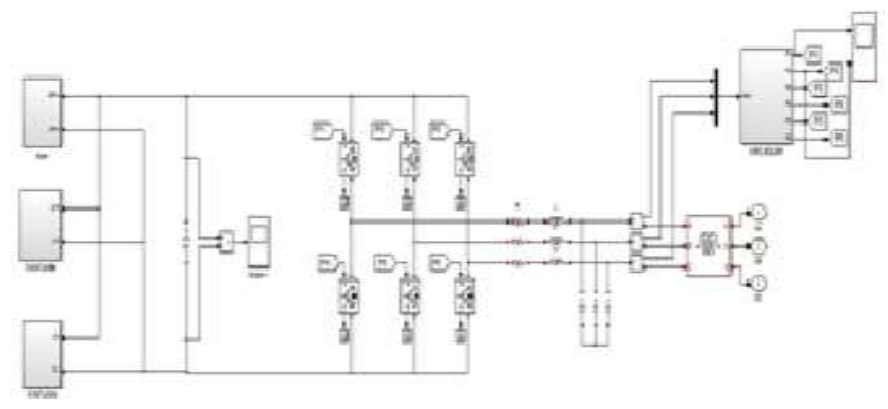

Figure 2.7. Predictive VSI model

In the above figure, incorporated model of all the DGs with VSI is shown. Due to the presence of non-linear loads in the Microgrid, especially in isolated mode of operation this new and efficient model is effective. Smart controller is also modeled with predictive technique which brings most reliable way in controlling the Microgrid technology. Control techniques for distributed energy sources based VSI depends on various factors like description of micro- energy and the concerned interface, intonation, merging and cache technology. The modulation method determines harmonic content of the Microgrid system at any instant.

Model predictive controls the voltage mode control with the variation of the duty cycle in square wave. Thyrsitor (IGBT) based VSI on PWM inverters are used for the reduction in damages and increased performance. In the case of filters, three phase LPF is installed for regularity output of inverter. Feedback loop can be carried out using predictive technique reference frames. For tracking the control requests, gate pulses are generated using power devices within the VSI. Custom power devices are used to eliminate the harmonics and compensate the reactive power by the injection of compensating currents and voltages at the PCC. Using a interconnection transformer, VSI usually integrated into the primary load bus. Power devices which implement efficient and good quality of power within DGs have been chosen [7].

\section{Interface Design}

Point of common coupling is place where the DGs are accommodated with main grid and loads using coupling inductors. In implementation for three phase balanced load as a series combination of RLC elements can be configured using Simpower Systems. Load block displays continuous impedance at specified frequency. At PCC the CPD like distribution static compensator are coupled using transformer with the help of predictive technique. Loads are connected into the load bus using static switches or inductors. Depending upon the type of compensators filters is also used at each and every points of PCC to smoothen the output from the each inverter. During islanded mode MSCC have to control the microgrid separately by generation of reference voltages for the proper integration to load bus. 


\section{SYSTEM INTEGRATION AND CONTROL}

Without the modification of the existing Microgrid infrastructure, design of control system with adds one of advanced sources. DGs can be connected or disconnected from the main grid more rapidly and smoothly, to meet the aggressive load demand frequency and power can be regulated independently. Response to the changes in the Microgrid system from DGs, without the requirement of programmable information must be inevitable. With the basic unity power factor controls, smart grid connection is not possible. Local voltage and frequency controls can't be able to control deviations due to the integration of DGs into the current system. In the attainment of local stability and reliability, zero current is been circulated within the Sources with necessary actions.

The real as well as reactive power of each micro-source is dependent with inverter output voltage developed. The active as well as reactive power at the PCC can be realized by

$$
\begin{aligned}
& P=\frac{3 E V \sin \delta}{\omega L} \\
& Q=\frac{3 V\{E \cos (\delta)-V\}}{\omega L}
\end{aligned}
$$

In which $\mathrm{V}$ and $\mathrm{E}$ are output voltages of grid and inverter respectively. $\mathrm{L}$ is the magnitude of inductive reactance. Controlling with VSI for both the magnitude and phase Consequently

$$
\begin{aligned}
& P=\frac{E V \sin \delta}{X} \\
& Q=\frac{V\{E \cos (\delta)-V\}}{X}
\end{aligned}
$$

Flow of real and reactive power is determined by the relationship between the inverter voltages $\mathrm{E}$, system voltage $\mathrm{V}$, and the inductor reactance $\mathrm{X}$ measured form the system.

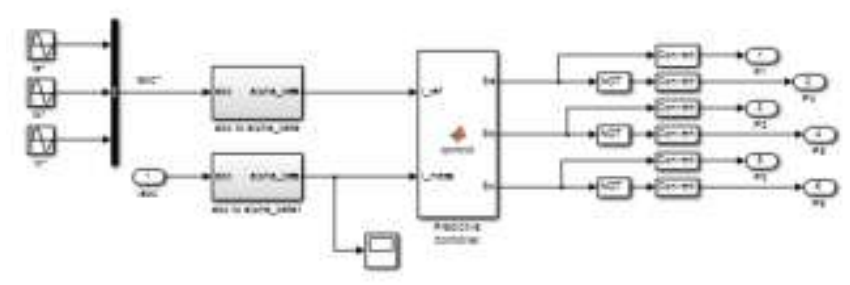

Figure 3.1. Predictive Model for Smart grid

The normal linear structure of MPC is displayed in the above figure. MPC begins regulating the control signals of the reference changes. It is possible to include NL predictive control into linear MPC and works reliably in isolated mode. The measured values of currents and voltages are given to the predictive model and power will be taken out after compensation. Due to prediction horizon method in addition to the reference signals are converted to alpha- beta version and produces compensating currents and voltages or either power and fed to the point of common coupling. A number of seven states are considered from $V_{0}$ to $V_{7}$ with initial and

final values are taken as zero. In the RH method, the different states are taken as below,

$$
\begin{aligned}
& V_{1}=\frac{2}{3} * V_{\mathrm{dc}} \cdots \\
& \mathbf{V}_{2}=\frac{1}{3} * \mathbf{V}_{\mathbf{d c}}+1 \mathbf{j} * \frac{\operatorname{sqrt}(3)}{3} * \mathbf{V}_{\mathbf{d c}} \cdots \\
& \mathbf{V}_{3}=-\frac{1}{3} * \mathbf{V}_{\mathbf{d c}}+1 \mathbf{j} * \frac{\operatorname{sqrt}(3)}{3} * \mathbf{V}_{\mathbf{d c}} \cdot-\cdot-\cdot \\
& \mathbf{V}_{4}=-\frac{2}{3} * \mathbf{V}_{\mathrm{dc}} \\
& \mathbf{V}_{\mathbf{5}}=-\frac{1}{3} * \mathbf{V}_{\mathbf{d c}}+1 \mathbf{j} * \frac{\operatorname{sqrt}(3)}{3} * \mathbf{V}_{\mathbf{d c}} \\
& \mathbf{V}_{6}=\frac{1}{3} * \mathbf{V}_{\mathbf{d c}}+1 \mathbf{j} * \frac{\operatorname{sqrt}(3)}{3} * \mathbf{V}_{\mathbf{d c}}
\end{aligned}
$$

The equations of the state $\mathrm{V}_{1}$ and $\mathrm{V}_{4}$ are the complex conjugates and also $\mathrm{V}_{2}, \mathrm{~V}_{3}$ and $\mathrm{V}_{5}, \mathrm{~V}_{6}$ are also the conjugates with the measured values of dc voltage. These states form a matrix which is as below.

$$
\mathrm{V}=\left[\mathrm{V}_{0}, \mathrm{~V}_{1}, \mathrm{~V}_{2}, \mathrm{~V}_{3}, \mathrm{~V}_{4}, \mathrm{~V}_{5}, \mathrm{~V}_{6}, \mathrm{~V}_{7}\right]
$$

In the subsequent condition reference current values at the sampling time $\mathrm{K}$ which is the current instant is considered and it is specified via the equation

$$
\mathbf{i}_{\mathbf{k}_{\text {ref }}}=\mathbf{I}_{\mathbf{r e f}(1)}+\mathbf{1} \mathbf{j} * \mathbf{I}_{\mathbf{r e f}(2)}
$$

The measured current values are taken at the same sampling time $\mathrm{K}$ and the current state at $\mathrm{K}^{\text {th }}$ time is given by the equation,

$$
\mathbf{i}_{\mathbf{k}}=\mathbf{I}_{-} \operatorname{mean}(\mathbf{1})+\mathbf{1} \mathbf{j} * \mathbf{I}_{-} \operatorname{mean}(2)
$$

Then the estimation of back emf is governed by the formula

$$
\begin{array}{r}
\mathbf{e}=\left[\mathbf{V}\left(\mathbf{x}_{\text {old }}\right)-\frac{\mathbf{L}}{\mathbf{T}_{\mathbf{s}}} * \mathbf{i}_{\mathbf{k}}-\left(\mathbf{R}-\frac{\mathbf{L}}{\mathbf{T}_{\mathbf{s}}}\right) * \mathbf{i}_{\text {old }}\right] \\
{\left[\mathbf{x}_{\text {old }}=1 \& \mathbf{i}_{\text {old }}=(\mathbf{0}+\mathbf{j 0})\right]}
\end{array}
$$

The measured current value is taken for the subsequent iteration

$$
\mathbf{i}_{\text {old }}=\mathbf{i}_{\mathrm{k}} \text { [present state] }
$$

The value of $\boldsymbol{I}$ varies from 0 to $\mathrm{k}+1$ and $\mathrm{V} \_01=\mathrm{V}$ (i). The predicted current value at the $\mathrm{k}+1$ instant is specified by the equation,

$$
\mathbf{i}_{\mathbf{k} 1}=\left(\mathbf{1}-\mathbf{R} * \frac{\mathbf{T}_{\mathbf{s}}}{\mathbf{L}}\right) * \mathbf{i}_{\mathbf{k}}+\frac{\mathbf{T}_{\mathbf{s}}}{\mathbf{L}\left(\mathbf{V}_{\mathbf{0 1}}-\mathbf{e}\right)}
$$

Where V_01 is the voltage at the next state, $\mathbf{E}$ signifies the emf function calculated earlier.

The CF of the respective system/device which is tyrannized by an equation which is below,

$$
\mathbf{G}=\operatorname{abs}\left(\operatorname{real}\left(\mathbf{i k}_{\text {ref }}-\mathbf{i}_{\mathbf{k} 1}\right)\right)+\operatorname{abs}\left(\mathbf{i m g}\left(\mathbf{i}_{\mathbf{k r e f}}-\mathbf{i}_{\mathbf{k} 1}\right)\right)
$$

Where abs represents the absolute value after the calculation with the sum of the real and imaginary terms of the reference and measured values. The CF relies on the constraints or the disturbances like faults, harmonics, sag or swells and it is represented by the equation given below, 


$$
\begin{gathered}
\left.\mathbf{g}<g_{-} o p t\right) \\
\mathbf{g o p t}_{\mathbf{o t}}=\mathbf{g} \\
\mathbf{x}_{\text {opt }}=\mathbf{i}
\end{gathered}
$$

The optimum value is stored in X_old and the optimal switching states are been selected as of the equations given below,

$$
\begin{aligned}
& S_{a}=\operatorname{state}\left(x_{\text {opt }}, 1\right) \\
& S_{b}=\operatorname{state}\left(x_{\text {opt }}, 2\right) \\
& S_{c}=\operatorname{state}\left(x_{\text {opt }}, 3\right)
\end{aligned}
$$

The optimum value is predicted and the switching states are given to the inverters as pulses at the gate terminal. The smart controller controls the power flows into and back from the Microgrid system by using the predictive technique.

When DGs operates in synchronized along with primary grid, loads are powered by both the sources. If grid lost power due to IEEE 1547 events, voltage drops, faults blackouts etc., Microgrid autonomously transfer to island mode. For dealing these types of faulty situations predictive control is the better method for deviations in voltage and frequency. The real time values obtained for the above frequency and voltages. For Inverters appropriate voltage tuning and frequency can be provided by MSCC.

\section{EXPERIMENTAL ANALYSIS RESULTS}

In the amalgamation of DER with grid, state transition numbers like 0 and 1 are assigned to all sources with an idea of source provides power take as 1 and 0 as power not available. Take an example, when all the three sources are appropriated in particular time, eight combinations are possible ie.000, 100, 110, 010, 011, 001,101 and 111.The availability in power and also status of supply which is available in Microgrid are represented by each sequence. 1 represents the ON state and 0 represents OFF state. Arrowhead represents the transition from On to OFF or vice versa like previous state to current state. Last digit gives the Grid and first two digits by DGs in each sequence.

The following case studies make us analyze the integration method:

\section{A. Case 1: Ordinary operation}

When Mode $\{101\}$ considered which gives DG1 = On, $\mathrm{DG} 2=\mathrm{OFF}$ and Grid $=\mathrm{ON}$, one $\mathrm{DG}$ alone provides power to the load on other side. Current operation gives the clear perception that, if load demand increases, a transition DG2 = ON takes place and Microgrid goes to full mode $\{111\}$. Presently both the grid and the DGs provide power to loads in the Microgrid. This type of operation is otherwise called as grid connected or synchronized mode. Consider now that the load demand decreases and DG is not that much efficient to provide power like Wind. In this scenario Microgrid will have a transition to $\{011\}$ mode to maintain stability and reliability. DG2 will keep on operating with the grid. This operation is shown bellow. predictive algorithm are used to implement the feedback for based predictive strategy can be implemented. Binary

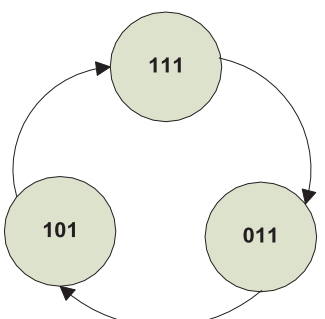

Figure4.1.Transitions and Control modes for Ordinary operation

\section{B. Case 2: Operation in Fault Condition}

In fault condition, there is non-availability of primary grid power. Therefore Grid $=$ OFF. Consider the state $\{010\}$ that corresponds to DG1 = OFF, DG2 = On. If in need for more power, the transition DG1- $\rightarrow$ On occurs and system transfers to the state $\{110\}$. Both of the DGs are supplying power in current case. When the load demand cuts down, the power from DG2 can be shut off and system will moves to the state $\{100\}$. So therefore DG1 will be used to supply the power to the loads. This transition is shown in figure 4.2. The transient response of the Microgrid is shown in the figure 4.3 .

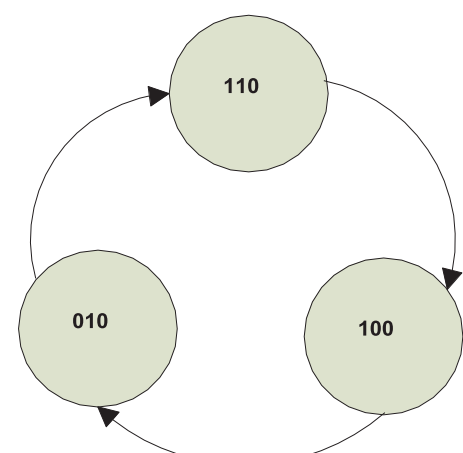

Figure 4.2. Strategy for Fault operation

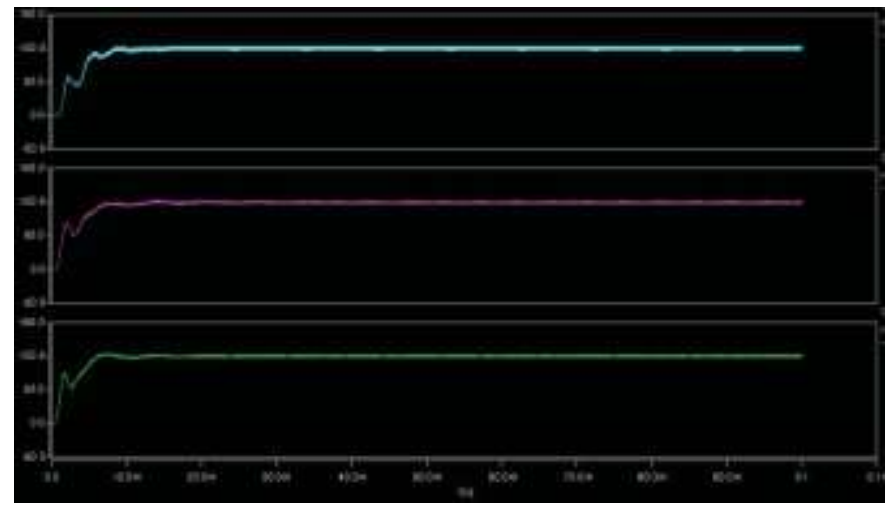

Figure 4.3. Transient response of Microgrid with faults

Execution of the transitions in a proper and efficient way controls the decisions that should be taken on the type of sources, must supply power according to the load requirement and factor of quality of power. Information regarding Grid status (ON and OFF time) is decided by the communication with the grid from the Microgrid point of view. That decides the backup power if available and with 
how many power sources. Transient conditions are verified and performance was analyzed in all the modes of operation. Simulation of recovery time has been performed on all conditions. The idea of the simulation time gives a clear aspect of operation and switching mode with transients and demand in load. This approach of predictive technique is resilient in the smart grid operations in normal and fault conditions of Microgrid.

\section{CONCLUSION}

An extensible scenario in combination or addition of distributed resources into the Microgrid via smart process is proposed in current paper. The idea of addressing seamless operation of Smart grid in spite of colossal renewable sources are been discussed. New complexities and architectures arise when there is an addition of sensing operation like predictive technique with sources, loads and utility operators. In order to gain high performance, economic cost and reliability advanced controlling techniques are incorporated for the realization of intelligent power system. This can be achieved only with the help of Predictive based Microgrid and smart grid. It needs lot of attention to bring and combine all this together into one homogenized system. The proposed method in present paper focus on all facets for development of smart microgrid structure, which is capable of performing automatic remedial action.

\section{REFERENCES}

1. Juan W. Dixon, Gustavo Venegas and Luis A. Moran, "A series Active Power Filter based on Sinusoidal Current controlled Voltage Source Inverter", IEEE Transactions on industrial Electronics, Vol.44, No.05, pp:612-620.

2. T. Devaraju, V. C. Veera reddyand M. Vijay Kumar, "Modeling and Simulation of Custom power devices to Mitigate Power quality Problems", International Journal of Engineering Science and Technology, Vol.26, 2012.

3. Shahid A " An Overview of control architecture for next generation smart grids", $19^{\text {th }}$ International Conference on intelligent System Applications to power, 2017.

4. Frede Blabjerg, "Power electronics -the key technology for renewable energy system integration", $4^{\text {th }}$ International Conference on Renewable Energy Research and Applications, 2015.

5. Gu, Y, Ouyang H, “ Development of Microgrid coordination and control Overview", IEEE PES innovative Smart Grid technologies, Asia, 2012.

6. Basu.M, Das, "Comparative Evaluation of two Models of UPQC for Suitable interface to enhance power quality", Electric Power systems Research, Vol.77, no.7, pp.821-830, 2007.

7. V. Khakikarand A., “ A new control Philosophy for a unified Power quality conditioner to coordinate load reactive power demand between shunt and series inverters", IEEE transactions on Power delivery, vol.23, no4, pp.2522- 2534, 2008.

8. N.G. Hingoroniand L. Gyugyi, Understanding FACTSConcepts and Technology of flexible AC Transmission Systems, IEEE Press , New York , 2000 